\title{
Spectral dimension with deformed spacetime signature
}

\author{
Jakub Mielczarek* and Tomasz Trześniewski*,† \\ ${ }^{*}$ Institute of Physics, Jagiellonian University, ul. Łojasiewicza 11, 30-348 Kraków, Poland \\ ${ }^{\dagger}$ Institute for Theoretical Physics, University of Wrocław, pl. Borna 9, 50-204 Wrocław, Poland
}

(Dated: July 21, 2017)

\begin{abstract}
Studies of the effective regime of loop quantum gravity (LQG) revealed that, in the limit of Planckian curvature scales, spacetime may undergo a transition from the Lorentzian to Euclidean signature. This effect is a consequence of quantum modifications of the hypersurface deformation algebra, which in the linearized case is equivalent to a deformed version of the Poincaré algebra. In this paper the latter relation is explored for the LQG-inspired hypersurface deformation algebra that is characterized by the above mentioned signature change.

While the exact form of the deformed Poincaré algebra is not uniquely determined, the algebra under consideration is representative enough to capture a number of qualitative features. In particular, the analysis reveals that the signature change can be associated with two symmetric invariant energy scales, which separate three physically disconnected momentum subspaces.

Furthermore, the invariant measure on momentum space is derived, which allows to properly define the average return probability, characterizing a fictitious diffusion process on spacetime. The diffusion is subsequently studied in the momentum representation for all possible variants of the model. Finally, the spectral dimension of spacetime is calculated in each case as a function of the scale parameter. In the most interesting situation the deformation is of the asymptotically ultralocal type and the spectral dimension reduces to $d_{S}=1$ in the UV limit.
\end{abstract}

\section{INTRODUCTION}

Analysis of a (fictitious) diffusion process on space or spacetime has become a versatile tool for characterizing classical and quantum models. The usefulness of this method stems from the spectral properties of Laplace operators, which allow us to determine such quantities as the return probability or spectral dimension. The former can be applied to calculations of the vacuum energy density and entanglement entropy [1]. Studies of the spectral dimension, which is one of the possible tools allowing to characterize the spacetime dimensionality at different scales, has become especially popular over the last ten years in the context of quantum gravity. In particular, this is so because the spectral dimension can tell us what is effective spacetime dimension perceived by a field defined of a given spacetime. The results may also be used for a comparison of different models of the Planck scale physics. However, the potential convergence of their predictions has to be treated as a hint rather than indication of the more fundamental relation. The reason is that different structures of (quantum) geometry may lead to similar running of the spectral dimension with scale.

The above mentioned avalanche of papers concerning the spectral dimension has been triggered by the seminal analysis performed in the framework of Causal Dynamical Triangulations (CDT) [2]. In the space of parameters of this model one can distinguish several phases, characterized by different geometrical properties. Deep within phase $\mathrm{C}$, in which one obtains an extended physical universe, it has been shown that spacetime undergoes a dimensional reduction from the classical value $d_{S} \approx 4$ at large diffusion times (IR limit) to $d_{S} \approx 2$ at small times (UV limit). The result has been subsequently generalized, showing that the UV value of the spectral dimen- sion varies depending on a position on the CDT phase diagram. In particular, in the region of the phase $\mathrm{C}$ close to the phase A (where spacetime becomes a sequence of short-living small universes) the value $d_{S} \approx 3 / 2$ has been measured, which, interestingly, can serve as a resolution to the entropic argument against the asymptotic safety scenario [3].

The dimensional reduction to $d_{U V}=2$ in the ultraviolet limit seems to be a common feature of a variety of approaches to quantum gravity [4]. It has been observed in (besides CDT): Hořava-Lifshitz gravity (for the characteristic exponent $z=3$ ) [5], asymptotic safety scenario [6], multi-fractal spacetimes 7], causal sets (probed by a scalar field) [8, 9] and spin-foam models [10, 11]. On the other hand, the value $d_{U V}=2$ is not completely universal but typical for a certain class of models, describing a specific type of quantum spacetime configurations, perhaps a specific phase of the gravitational field. In particular, as we already mentioned, the values different from 2 can be found on the CDT phase diagram. While in the physical phase $\mathrm{C}$ the dimensional reduction from $d_{I R}=4$ to $d_{U V} \in[3 / 2,2]$ has been observed, in two other phases values of the spectral dimension are significantly different, e.g. in the non-geometric phase B (where universe is a highly-connected single time slice) the spectral dimension diverges at small scales. Another example of $d_{U V} \neq 2$ is provided by the $\kappa$-Minkowski noncommutative spacetime, which is often employed in models of doubly special relativity or relative locality, characterized by deformed relativistic symmetries. It has been shown to exhibit the dimensional reduction from $d_{I R}=4$ to $d_{U V}=312$ but this value is obtained for a particular choice of the Laplace operator on momentum space. Meanwhile, in the case of the relative locality-inspired Laplacian the spectral dimension diverges at small scales [13]. One of the 
issues in the above context is also the interplay between the running dimension and either breaking or deforming of the relativistic symmetries of spacetime 14].

In this paper we extend previous results by investigating the diffusion on spacetime whose Poincaré symmetries are deformed by effects predicted in the effective regime of loop quantum gravity (LQG). Namely, it has been shown that as a consequence of requiring the anomaly freedom the classical algebra of gravitational constraints (the hypersurface deformation algebra) is subject to a quantum deformation [15, 16]. A pronounced manifestation of the deformation is the phenomenon of a "dynamical" signature change [16 18]. Here, in particular, we study the role of such a signature change in the description of a diffusion process. Our calculations are performed in the symmetry-reduced setup, where the isotropy and homogeneity of spacetime are assumed. Then the hypersurface deformation algebra is equivalent to a deformed version of the Poincaré algebra. The explicit form of the latter deformation has so far been obtained only in the spherically symmetric case [19], where the algebra is effectively two-dimensional. The corresponding deformed Poincaré algebra (and, tentatively, the coalgebra) was also studied in $20-22]$. An additional effect of such investigations is that they provide a desired prediction of the deformed relativistic symmetries from the hypothetical full theory of quantum gravity, while usually they have only been introduced a priori, in the phenomenological approach 23]. Finally, let us mention that in [22] it has been suggested that the loop deformation of the hypersurface deformation algebra may lead to the dimensional reduction to $d_{U V}=2.5$ near the Planck scale. However, this value can change when higher order contributions to the dispersion relation are taken into account.

In the next sections, inspired by the analysis performed in 24], we recover the form of a particular deformed four-dimensional Poincaré algebra by imposing certain reasonable conditions. While such a method does not lead to the unique form of the deformation, the obtained results allow to deduce a number of qualitative conclusions. In particular, as we show, the deformation may lead to the appearance of a new invariant energy scale. Furthermore, we explicitly derive the invariant measure on momentum space, which turns out to be different from the one that could be naively expected from the form of the mass Casimir. The symmetry algebra turns out to be compatible with the standard Heisenberg algebra of phase space variables and therefore we assume that the latter algebra is undeformed. Finally, (in the momentum representation) we calculate the average return probability and the resulting spectral dimension of spacetime, which is a function of the scale parameter. We analyze four distinct cases, depending on the deformation parameter. The physical meaning and relations of the results with other approaches are discussed.

Throughout this paper we use the Planck units, where $\hbar=c=1, G=1 / m_{\mathrm{Pl}}^{2}$ and $m_{\mathrm{Pl}}$ denotes the Planck mass.

\section{DEFORMED POINCARÉ ALGEBRA}

The perturbative analysis of the effective regime of loop quantum gravity has revealed (see e.g. [15-17]) the following form of the quantum-deformed hypersurface deformation algebra:

$$
\begin{aligned}
\left\{D\left[N_{1}^{a}\right], D\left[N_{2}^{a}\right]\right\} & =D\left[N_{1}^{b} \partial_{b} N_{2}^{a}-N_{2}^{b} \partial_{b} N_{1}^{a}\right] \\
\left\{S[N], D\left[N^{a}\right]\right\} & =-S\left[N^{b} \partial_{b} N\right] \\
\left\{S\left[N_{1}\right], S\left[N_{2}\right]\right\} & =D\left[s \Omega q^{a b}\left(N_{1} \partial_{b} N_{2}-N_{2} \partial_{b} N_{1}\right)\right]
\end{aligned}
$$

where $q^{a b}$ denotes the spatial metric, with spatial indices $a, b=1,2,3$, and $s$ is the spacetime metric signature. $D\left[N^{a}\right]$ is the constraint generating spatial diffeomorphisms, parametrized by a shift vector field $N^{a}$, while $S[N]$ is the scalar constraint and generator of deformations in the direction normal to spatial hypersurfaces, parametrized by a lapse function $N$. Due to the effects of LQG the scalar constraint is subject to a quantum deformation. Moreover, the hypersurface deformation algebra itself is deformed through the presence of the factor $\Omega$, which is some function of gravitational field variables. The general form of this function in LQG is not yet known but it has been explicitly derived for specific symmetry reduced configurations. In the case when the sign of $\Omega$ is constant the deformation can actually be absorbed by an appropriate transformation of variables, which cannot be achieved if the sign is changing [25].

In particular, in the case of a homogeneous and isotropic spacetime configuration on which there are introduced perturbative inhomogeneities with holonomy corrections the deformation factor is given by [16]

$$
\Omega=\cos (2 \gamma \bar{\mu} \bar{k}) \cong 1-2 \frac{\rho}{\rho_{c}} \in[-1,1] .
$$

Here $\cong$ denotes imposition of the constraint $S[N], \bar{p}$ and $\bar{k}$ are the homogeneous Ashtekar variables, $\gamma \sim 1$ is the Barbero-Immirzi parameter and $\bar{\mu}=\sqrt{\Delta / \bar{p}}$ the lattice refinement, with $\Delta$ being the minimal area, expected to be of the order of the Planck area $\Delta \sim 1 / m_{\mathrm{Pl}}^{2} \cdot \rho$ is energy density of the matter content of universe and $\rho_{c}=3 m_{\mathrm{Pl}}^{2} /(8 \pi \gamma \Delta) \sim m_{\mathrm{Pl}}^{4}$ the maximal allowed value of energy density, expected to be of the order of the Planck density. We note that the classical value $\Omega=1$ is correctly recovered in the limit of low energy densities $\rho \rightarrow 0$. On the other hand, when the density reaches the maximum $\rho \rightarrow \rho_{c}$, the opposite value $\Omega=-1$ is achieved.

Since the deformation factor $\Omega$ in (3) is accompanied by the metric signature $s$, the change of the sign of $\Omega$ can be interpreted as the signature change [18. Namely, at low energy densities we have $s \Omega \rightarrow s$, which corresponds to the classical Lorentzian (for $s=1$ ) or Euclidean (for $s=-1)$ space, while at the maximal density $\rho=\rho_{c}$, in the deep quantum regime, the effective signature becomes $s \Omega \rightarrow-s$. Moreover, at $\rho=\rho_{c} / 2$ the sign of $s \Omega$ turns out to be indefinite (i.e. $s \Omega=0$ ), which can be associated with the state of so-called asymptotic silence or ultralocality [26]. 
In this paper we are going to consider the algebra (1. (3) restricted to linear hypersurface deformations, characterizing homogeneous and isotropic spacetime configurations. In such a case the above deformed algebra of constraints (for $s=1$ ) is equivalent to a certain deformation of the Poincaré algebra, by which we mean the universal enveloping algebra of a Lie algebra $\mathfrak{i s o}(3,1)$.

As we discuss it in Appendix, the limit of linear deformations can be imposed on (1, 3) by choosing the spatial metric to be given by the Kronecker delta, $q_{a b}=\delta_{a b}$ and restricting the expressions for a lapse function and shift vector to the linear form:

$$
N(x)=\Delta t+v_{a} x^{a}, \quad N^{a}(x)=\Delta x^{a}+R_{b}^{a} x^{b},
$$

where $\Delta t, \Delta x^{a}, v_{a}$ and $R^{a}{ }_{b}$ are the infinitesimal parameters of, respectively, a time translation, spatial translations, boosts and rotations. The rotation matrix can be expressed in terms of infinitesimal angles $\varphi^{a}$ as $R^{a b}=$ $\epsilon^{b a c} \varphi_{c}$. Under such assumptions the scalar and diffeomorphism constraints can be expanded into the following combinations of the Poincaré algebra generators:

$$
\begin{aligned}
S[N] & =S\left[\Delta t+v_{a} x^{a}\right]=-\Delta t P_{0}-v^{a} K_{a}, \\
D\left[N^{a}\right] & =D\left[\Delta x^{a}+R^{a}{ }_{b} x^{b}\right]=-\Delta x^{b} P_{b}-\varphi^{b} J_{b} .
\end{aligned}
$$

where $P_{0}, P_{a}, J_{a}$ and $K_{a}$ denote the generators of time translations, spatial translations, boosts and rotations, respectively.

In Appendix we show how the (standard) Poincaré algebra can be recovered by imposing the conditions $q_{a b}=\delta_{a b}$ and (6-7) on the classical hypersurface algebra. The loop deformed algebra (1][3), although very similar to the classical one, does not allow to straightforwardly apply the same methodology. While for the undeformed brackets (112) the derivation of the corresponding sector of the Poincaré algebra is the same as in Appendix, the bracket (3) contains the additional function $\Omega$ inside the diffeomorphism constraint. The most convenient solution would be to find a way to extract $\Omega$ in front of the constraint. However, apart from the perturbative approach, which has been used e.g. in deriving (4), it is not possible to do so directly.

The strategy that we are going to apply here is based on the observation that a diffeomorphism constraint with some additional function $f$ of field variables inside can always be rewritten as the unmodified diffeomorphism constraint multiplied by a certain functional of $f$. Namely,

$$
D\left[f N^{a}\right]=F[f] D\left[N^{a}\right],
$$

where the functional $F[f]$ is simply given by

$$
F[f]=\frac{D\left[f N^{a}\right]}{D\left[N^{a}\right]}=:\langle f\rangle_{D},
$$

which can be interpreted as the diffeomorphism average of the function $f$. Then the problematic right hand side of (3) can be expressed as

$$
\begin{aligned}
& D\left[s \Omega q^{a b}\left(N_{1} \partial_{b} N_{2}-N_{2} \partial_{b} N_{1}\right)\right] \\
& =s\langle\Omega\rangle_{D} D\left[q^{a b}\left(N_{1} \partial_{b} N_{2}-N_{2} \partial_{b} N_{1}\right)\right] \\
& =-s_{\mathrm{eff}}\left(v^{a} P_{a}+\varphi^{a} J_{a}\right),
\end{aligned}
$$

where we introduce the effective metric signature

$$
\begin{aligned}
s_{\mathrm{eff}}=s \tilde{\Omega} & :=s\langle\Omega\rangle_{D} \\
& =s \frac{D\left[\Omega q^{a b}\left(N_{1} \partial_{b} N_{2}-N_{2} \partial_{b} N_{1}\right)\right]}{D\left[q^{a b}\left(N_{1} \partial_{b} N_{2}-N_{2} \partial_{b} N_{1}\right)\right]} .
\end{aligned}
$$

The quantity $s_{\text {eff }}$ has been called "effective signature" since it reduces to the metric signature $s$ in the classical limit, while otherwise it is instead a certain function of the Poincaré algebra generators. Because $s_{\text {eff }}$ multiplies the $P_{a}$ and $J_{a}$ generators in (10) in the same way as signature $s$ in the classical case, it is possible to expand the bracket (3) analogously to its classical counterpart (80). Consequently, using the procedure discussed in Appendix we obtain the deformed Poincaré algebra that is determined by the following brackets:

$$
\begin{aligned}
\left\{J_{a}, J_{b}\right\} & =\epsilon_{a b c} J^{c}, \\
\left\{J_{a}, K_{b}\right\} & =\epsilon_{a b c} K^{c}, \\
\left\{K_{a}, K_{b}\right\} & =-s_{\mathrm{eff}} \epsilon_{a b c} J^{c}, \\
\left\{J_{a}, P_{b}\right\} & =\epsilon_{a b c} P^{c}, \\
\left\{J_{a}, P_{0}\right\} & =0, \\
\left\{K_{a}, P_{b}\right\} & =\delta_{a b} P_{0}, \\
\left\{K_{a}, P_{0}\right\} & =s_{\mathrm{eff}} P_{a}, \\
\left\{P_{a}, P_{b}\right\} & =0, \\
\left\{P_{a}, P_{0}\right\} & =0,
\end{aligned}
$$

where the effective signature $s_{\text {eff }}=s \tilde{\Omega}$ appears only where $s$ is located in the standard Poincaré algebra. As one can see, $\tilde{\Omega}$ is some unknown function of the symmetry generators, related via (11) to the deformation factor $\Omega$, which is present at the level of the hypersurface deformation algebra. If the form of $\Omega$ was known as a function of field variables, then one could also try to derive the form of $\tilde{\Omega}$, using e.g. the Brown-York generators [27].

Precisely speaking, our deformed Poincaré algebra is a certain quotient of the tensor algebra defined by the brackets (12,20) and the latter is actually a semi-classical deformation of a Poisson algebra. In other words, $P_{0}$, $P_{a}, J_{a}$ and $K_{a}$ can be viewed as functions on classical phase space (spacetime and the corresponding momentum space) but satisfying the deformed brackets. Passing to the quantum theory would require replacing $\{\cdot, \cdot\} \longrightarrow \frac{1}{i}[\cdot, \cdot]$ and promoting $P_{0}, P_{a}, J_{a}$ and $K_{a}$ to quantum operators. However, for the purposes of this paper it is not necessary to discuss the full quantum picture (in particular, in the framework of quantum groups) and therefore we restrict the considerations to the semiclassical regime.

As mentioned above, a priori we do not know the functional form of the deformation factor $\tilde{\Omega}$. However, we are 
able to constrain it using mathematical consistency and experience gained from the symmetry reduced models, as well as calculate it explicitly in some particular cases. Let us first note that since $\tilde{\Omega}$ is generally a non-trivial function of the algebra generators, the brackets (12) 200 do not define a Lie algebra. Nevertheless, it is reasonable to require the Jacobi identities, which are a feature of the Poincaré (Lie) algebra, to be satisfied even for a deformed version of this algebra. The main motivation to do so is that in such a case the (standard) Leibnitz rule for the action of an algebra on itself is preserved. One can also naturally expect that $\tilde{\Omega}$ is rotationally invariant. Moreover, the ordinary Poincaré algebra should be recovered in the limit of vanishing deformation (which will correspond to low energies). Our final assumption, which we choose here as a specific simple example, is that $\tilde{\Omega}$ depends only on the translation generators and is a separable function of the form $\tilde{\Omega}=\tilde{\Omega}\left(P_{0}, \mathbf{P}^{2}\right)=A\left(P_{0}\right) B\left(\mathbf{P}^{2}\right)$, where $A$ and $B$ can be determined from the other conditions. If we allowed $\tilde{\Omega}$ to depend on rotations $J_{a}$ and boosts $K_{a}$, the analysis and interpretation of results would be much more difficult.

In the case of Lorentzian signature $s=1$ the Jacobi identities lead to a differential equation on $\tilde{\Omega}=$ $\tilde{\Omega}\left(P_{0}, \mathbf{P}^{2}\right)$, which has the solution

$$
\tilde{\Omega}\left(P_{0}, \mathbf{P}^{2}\right)=\frac{P_{0}^{2} / c_{1}-1}{\mathbf{P}^{2} / c_{2}-1},
$$

with two independent real constants $c_{1}, c_{2}$. As we will now show, the classical limit imposes a relation between $c_{1}$ and $c_{2}$.

By construction, (21) commutes with all generators of the algebra (12) and hence it is a Casimir element of the latter. Then the mass Casimir element $\mathcal{C}_{1}$ of the algebra (determining the mass square of a particle whose symmetries are described by (12|20), with the standard classical limit, can be constructed as the appropriate combination of $\tilde{\Omega}$ and the unit element, namely

$$
\mathcal{C}_{1}=a_{1} \tilde{\Omega}+a_{2} \mathbb{I}=\frac{-\frac{a_{1}}{c_{1}} P_{0}^{2}-\frac{a_{2}}{c_{2}} \mathbf{P}^{2}+\left(a_{1}+a_{2}\right)}{1-c_{2}^{-1} \mathbf{P}^{2}} .
$$

The conditions that have to be satisfied in order to recover the proper classical limit are $a_{2}=-a_{1}, a_{1}=c_{1}$ and $c_{1}=c_{2} \equiv \alpha$, where $\alpha$ is a (positive or negative) real constant. Consequently, the mass Casimir can be written as 24.

$$
\mathcal{C}_{1}=\frac{-P_{0}^{2}+\mathbf{P}^{2}}{1-\alpha^{-1} \mathbf{P}^{2}}
$$

and the classical expression is recovered for $|\alpha| \rightarrow \infty$.

Furthermore, taking into account the fact that $c_{1}=$ $c_{2} \equiv \alpha$, the deformation factor (21) simplifies to

$$
\tilde{\Omega}\left(P_{0}, \mathbf{P}^{2}\right)=\frac{P_{0}^{2}-\alpha}{\mathbf{P}^{2}-\alpha} .
$$

Let us note that, in contrast to (4), $\tilde{\Omega}$ is not a bounded quantity. In the calculations we assumed that in the limit $|\alpha| \rightarrow \infty$ the expression (24) tends to 1 , restoring the undeformed Poincaré algebra. Below we will extend the symmetry algebra by the Heisenberg algebra and identify the translation generators with components of a momentum. Then (24) can also be seen as tending to 1 in the low energy limit $P_{0} \rightarrow 0, \mathbf{P} \rightarrow 0$.

Let us now consider an extension of the symmetry algebra (12-20) by the (undeformed) Heisenberg algebra of spacetime positions $x_{\mu}$ and four-momenta $p_{\mu}$, defined by the brackets

$$
\left\{x_{\mu}, x_{\nu}\right\}=0, \quad\left\{x_{\mu}, p_{\nu}\right\}=\eta_{\mu \nu}, \quad\left\{p_{\mu}, p_{\nu}\right\}=0,
$$

with spacetime indices $\mu, \nu=0,1,2,3$ and where $\eta=$ $\operatorname{diag}(-1,1,1,1)$ is the Minkowski metric. Applying the approach of [28] we find the following realization of [12. 20) in terms of the $x_{\mu}$ and $p_{\mu}$ generators:

$$
\begin{aligned}
\epsilon_{a b c} J^{c} & =x_{a} p_{b}-x_{b} p_{a}, \\
K_{a} & =x_{a} p_{0}-x_{0} p_{a} \tilde{\Omega}, \\
P_{a} & =p_{a}, \quad P_{0}=p_{0} .
\end{aligned}
$$

The above expressions do not depend on the explicit form of $\tilde{\Omega}=\tilde{\Omega}\left(P_{0}, P_{a}\right)$. Then for $\tilde{\Omega}$ given by (24) we find that the brackets of $x_{\mu}$ with the boost generators have the non-trivial form

$$
\begin{aligned}
& \left\{K_{a}, x_{0}\right\}=x_{a}-2 x_{0} \frac{p_{0} p_{a}}{p_{0}^{2}-\alpha} \tilde{\Omega}, \\
& \left\{K_{a}, x_{b}\right\}=x_{0} \tilde{\Omega}\left(\delta_{a b}-2 \frac{p_{a} p_{b}}{\mathbf{p}^{2}-\alpha}\right)
\end{aligned}
$$

and satisfy all necessary Jacobi identities. In this sense our deformed Poincaré algebra is compatible with the standard Heisenberg algebra (25). The merger of these two algebras describes usual commutative spacetime and corresponding momentum space but endowed with deformed relativistic symmetries. However, such an extension of the symmetry algebra is ambiguous, since the latter alone is actually insufficient to determine the form of the Heisenberg algebra. To this end we need also the coproduct and antipode on the algebra (12,20), which would turn the latter into a Hopf algebra. Then the appropriate Heisenberg algebra could generally be constructed using the so-called smash product construction, see e.g. [29]. Therefore, in principle, (12 20) can as well describe symmetries of a noncommutative spacetime, determined by some deformed Heisenberg algebra. Currently it is not yet known how the Hopf algebraic structure of symmetries can be extracted from the loop quantization in $3+1$ dimensions, apart from assuming a compatible hypothesis [20] (although it has recently been done in $2+1$ dimensions [30]). Therefore (25) should be understood as the simplest possible Ansatz for the Heisenberg algebra consistent with (12,20), which does not diverge too far from usual physics. 


\section{INVARIANT ENERGY SCALE}

The mass Casimir element (23) is invariant under rotations, since it is constructed from the deformation factor (24). Not surprisingly, the set of its symmetries is actually larger, since (23) is also preserved by an appropriate deformed version of the Lorentz transformations, which we will now introduce.

For simplicity let us restrict to boosts with a velocity $v$ in the $x$ direction. Then the deformed Lorentz transformation of a four-momentum $\left(P_{0},\left\{P_{a}\right\}\right)$ (whose components are identified with the translation generators via (28)) has the following form:

$$
\begin{aligned}
& P_{0}^{\prime}=Q \gamma\left(P_{0}-v P_{1}\right), \\
& P_{1}^{\prime}=Q \gamma\left(P_{1}-v P_{0}\right), \\
& P_{2}^{\prime}=Q P_{2}, \\
& P_{3}^{\prime}=Q P_{3} .
\end{aligned}
$$

The difference with respect to the ordinary transformations is contained in the factor

$$
Q=\frac{1}{\sqrt{1+\frac{\gamma^{2} v^{2}}{\alpha}\left(P_{0}^{2}+P_{1}^{2}-\frac{2}{v} P_{0} P_{1}\right)}}
$$

where $\gamma=\frac{1}{\sqrt{1-v^{2}}}$ is the usual Lorentz factor. In the classical limit $|\alpha| \rightarrow \infty$ we correctly obtain $Q \rightarrow 1$.

A natural consequence in this context is the existence of an energy scale that is invariant under the action of transformations (31, 34). Indeed, solving the equation $P_{0}^{\prime}=P_{0}$ for $P_{0}$ we find that it has two (real-valued) solutions $\pm \sqrt{\alpha}$, although only when $\alpha>0$. In other words, if we take an arbitrary four-momentum of the form $\left(P_{0}= \pm \sqrt{\alpha}, P_{1}, P_{2}, P_{3}\right)$ (with $\alpha>0$ ), then acting with a deformed boost (31) 34) we obtain the vector $\left(P_{0}^{\prime}, P_{1}^{\prime}, P_{2}^{\prime}, P_{3}^{\prime}\right)=\left( \pm \sqrt{\alpha}, Q \gamma\left(P_{1} \mp \sqrt{\alpha} v\right), Q P_{2}, Q P_{3}\right)$, which shows that the energy component $P_{0}$ is conserved. Therefore $\pm \sqrt{\alpha}$ are the invariant energy scales that we were looking for. Meanwhile, in the case of $\alpha<0$ such an invariant is purely imaginary and therefore we do not consider it as physical.

Let us note that $\pm \sqrt{\alpha}, \alpha>0$ are distinguished values of energy within our model for other reasons as well. Namely, both the deformation factor $\tilde{\Omega}$ and mass Casimir $\mathcal{C}_{1}$ become divergent at $|\mathbf{P}|=\sqrt{\alpha}$. Moreover, $\tilde{\Omega}$ changes sign when crossing either $|\mathbf{P}|=\sqrt{\alpha}$ or $\left|P_{0}\right|=\sqrt{\alpha}$. On the other hand, for $\alpha<0$ we observe that $\tilde{\Omega}$ is positive definite, there is no divergence of $\mathcal{C}_{1}$ and hence no distinguished energy scale. It is also worth to mention that some features of the invariant energy scale $\pm \sqrt{\alpha}$ are similar to the invariant scale characterizing one of the models of doubly special relativity [31.

Furthermore, it can be expected that the regions in four-momentum space determined by the energy values $\pm \sqrt{\alpha}-$ with $P_{0} \in(-\infty,-\sqrt{\alpha}), P_{0} \in(-\sqrt{\alpha}, \sqrt{\alpha})$ or $P_{0} \in(\sqrt{\alpha}, \infty)$ - are physically disconnected. More precisely, we want to check whether it is possible to boost a momentum from one region to another. To this end let us consider an arbitrary vector of the form $\left(P_{0}=\varepsilon \sqrt{\alpha}, P_{1}, P_{2}, P_{3}\right)$, where $\varepsilon \in(-1,1)$, so that $P_{0} \in(-\sqrt{\alpha}, \sqrt{\alpha})$. A deformed boost with the velocity $v$ transforms the energy component into

$$
P_{0}^{\prime}=\frac{\sqrt{\alpha}\left(\varepsilon \sqrt{\alpha}-v P_{1}\right)}{\sqrt{\left(\varepsilon \sqrt{\alpha}-v P_{1}\right)^{2}+\left(1-v^{2}\right)\left(1-\varepsilon^{2}\right) \alpha}},
$$

where naturally always $\left(1-v^{2}\right)\left(1-\varepsilon^{2}\right) \alpha>0$ and hence $-\sqrt{\alpha}<P_{0}^{\prime}<\sqrt{\alpha}$. Therefore energy remains in the range $(-\sqrt{\alpha}, \sqrt{\alpha})$, unless we take superluminal velocities. Choosing $\varepsilon \in( \pm 1, \pm \infty)$ one can reach the analogous conclusions for momenta with $P_{0}$ lying above $\sqrt{\alpha}$ or below $-\sqrt{\alpha}$. These three regions can be, therefore, described as physically separated momentum subspaces.

Lastly, let us briefly explore the issue of allowed velocities of particles characterized by the considered deformed symmetries. To this end we may consider the mass Casimir (23), which gives us the following dispersion relation for particles with mass $m$ :

$$
P_{0}^{2}=m^{2}+\mathbf{P}^{2}\left(1-\frac{m^{2}}{\alpha}\right)
$$

leading to the following relation between the phase and group velocities:

$$
v_{\mathrm{gr}} v_{\mathrm{ph}}=1-\frac{m^{2}}{\alpha}
$$

This allows us to express the group velocity as

$$
v_{\mathrm{gr}}=\frac{1-\frac{m^{2}}{\alpha}}{\sqrt{1-\frac{m^{2}}{\alpha}+\frac{m^{2}}{\mathbf{P}^{2}}}} .
$$

The maximal allowed value of the group velocity is obtained for $\mathbf{P}^{2} \rightarrow \infty$ and amounts to

$$
v_{\mathrm{gr}}^{\max }=\sqrt{1-\frac{m^{2}}{\alpha}} .
$$

Depending on the sign of $\alpha, v_{\mathrm{gr}}^{\max }$ can be smaller or greater than the speed of light in vacuum. However, for typical masses of particles the difference is expected to be very small. Even for the inflaton field, for which $m \sim 10^{-6} m_{\mathrm{Pl}}$, assuming $|\alpha| \sim m_{\mathrm{Pl}}^{2}$ one obtains the correction of the order $\frac{m^{2}}{|\alpha|} \sim 10^{-12}$. The effect would be, therefore, extremely difficult to observe. Moreover, for photons the dispersion relation takes the standard form and $v_{\mathrm{gr}}^{\max }=1$. Anyway, superluminal velocities are excluded in the case of $\alpha>0$.

\section{INVARIANT MEASURE}

When four-momentum space is endowed with a given algebra of symmetries, then the latter determines the 
form of the infinitesimal invariant volume element, which plays the role of a measure on this momentum space. For the Poincaré algebra the invariant momentum space measure is simply $d^{4} P$. On the other hand, since the symmetry algebra 120 and the corresponding transformations (31]34) are a deformed counterpart of the Poincaré case, one can reasonably expect that the invariant measure on momentum space with the symmetries $(12-20)$ is an appropriate modification of $d^{4} P$.

In order to explore this issue we calculate the Jacobian determinant of a momentum transformation (31-34)

$$
\operatorname{det}\left(\frac{\partial P_{\mu}^{\prime}}{\partial P_{\nu}}\right)=\left|\begin{array}{llll}
\frac{\partial P_{0}^{\prime}}{\partial P_{9}} & \frac{\partial P_{0}^{\prime}}{\partial P_{1}} & \frac{\partial P_{0}^{\prime}}{\partial P_{2}} & \frac{\partial P_{0}^{\prime}}{\partial P_{3}} \\
\frac{\partial P_{1}^{\prime}}{\partial P_{0}} & \frac{\partial P_{1}^{\prime}}{\partial P_{1}} & \frac{\partial P_{1}^{\prime}}{\partial P_{2}} & \frac{\partial P_{1}^{\prime}}{\partial P_{3}} \\
\frac{\partial P_{2}^{\prime}}{\partial P_{9}} & \frac{\partial P_{2}^{\prime}}{\partial P_{1}} & \frac{\partial P_{2}^{\prime}}{\partial P_{2}} & \frac{\partial P_{2}}{\partial P_{3}} \\
\frac{\partial P_{3}^{\prime}}{\partial P_{0}} & \frac{\partial P_{3}^{\prime}}{\partial P_{1}} & \frac{\partial P_{3}^{3}}{\partial P_{2}} & \frac{\partial P_{3}^{\prime}}{\partial P_{3}}
\end{array}\right|=Q^{6} .
$$

The above result leads to the following relation between the measure in the initial coordinates and the one in the boosted coordinates:

$$
d^{4} P^{\prime}=Q^{6} d^{4} P
$$

where $P=\left(P_{0},\left\{P_{a}\right\}\right)$ and $P^{\prime}=\left(P_{0}^{\prime},\left\{P_{a}^{\prime}\right\}\right)$.

Let us now try to find such a function $f(P)$ that the condition

$$
f\left(P^{\prime}\right) d^{4} P^{\prime}=f(P) d^{4} P,
$$

is satisfied. From (42) we infer that under a deformed boost (31) 34) the function $f(P)$ has to transform as

$$
f(P)=Q^{6} f\left(P^{\prime}\right) .
$$

Furthermore, the correspondence with the classical case requires that in the limit $|\alpha| \rightarrow \infty$ we have $f(P) \rightarrow 1$. Then the form of $f(P)$ can be deduced by observing that there exists the equality

$$
Q^{2}\left(1-\frac{\mathbf{P}^{2}}{\alpha}\right)=1-\frac{\mathbf{P}^{\prime 2}}{\alpha} .
$$

Combining all above relations we find the invariant momentum space measure

$$
d \mu \equiv \frac{f(P) d^{4} P}{(2 \pi)^{4}}=\frac{1}{\left(1-\frac{\mathbf{P}^{2}}{\alpha}\right)^{3}} \frac{d^{4} P}{(2 \pi)^{4}} .
$$

This result will be crucial for calculating the average return probability in Sec. VI.

It is worth stressing that the measure (46) differs from the one that could be naively expected from the mass Casimir (23). Namely, writing (23) as

$$
\mathcal{C}_{1}=g^{\mu \nu}(P) P_{\mu} P_{\nu}, \quad g^{\mu \nu}(P) \equiv \frac{\eta^{\mu \nu}}{1-\alpha^{-1} \mathbf{P}^{2}},
$$

one naturally deduces that the invariant measure should have the form

$$
\sqrt{\left|\operatorname{det}\left(g_{\mu \nu}(P)\right)\right|} d^{4} P=\left(1-\frac{\mathbf{P}^{2}}{\alpha}\right)^{2} d^{4} P .
$$

However, the measure obtained in such a heuristic way is explicitly breaking the invariance with respect to the deformed boosts (31, 34).

\section{EUCLIDEAN DOMAIN}

So far we have focused on the Lorentzian model, with the signature $s=1$. However, to introduce a diffusion process on spacetime we actually need to consider the Euclidean version of our deformed symmetry algebra. One of the issues in this context is that the Laplace operator defined on spacetime (as well as in the momentum space representation) has to be elliptic. For the model considered in this paper the situation is complicated by the fact that the sign of $\tilde{\Omega}$ changes with momentum. Therefore, we expect the Laplace operator to be of a mixed type (either elliptic or hyperbolic, depending on the energy range). In order to be able to probe spacetime by diffusion from large scales up to small scales we restrict here to the situation where spacetime is Euclidean at large scales. Nevertheless, one has to keep in mind that when $\alpha>0$ the sign of the Euclidean version of $\tilde{\Omega}$ changes and spacetime becomes Lorentzian for 3-momenta exceeding $\sqrt{\alpha}$, which is making the diffusion ill defined for such a regime of momenta. The alternative possibility would be to consider the Lorentzian case discussed in Sec. III again with the sign changing $\tilde{\Omega}$. Such a case is, however, also ill defined (for $\alpha>0$ ) since it would contain the regime where the measure is negative, leading to a substantial difficulty in interpreting the trace of heat kernel as the average return probability (see Sec. (VI). The latter could be remedied [32] by an appropriate modification of the diffusion equation but here we follow the conservative approach.

A transition from the Lorentzian to Euclidean domain can be performed either by an analytic continuation, called the Wick rotation, or by introducing a priori the Euclidean counterpart of the deformed Poincaré algebra. Since the analytic continuation may turn out to be tricky, as it is the case for the $\kappa$-Poincaré algebra (where the deformation parameter $\kappa$ has to be analytically continued as well, although this is so due to the coalgebra [33]), both of the possibilities are discussed below.

The Wick rotation that we consider here is the analytic continuation $P_{0} \longrightarrow-i P_{0}, K_{a} \longrightarrow-i K_{a}$. Applying it to the deformed brackets (12) 200 we find that the only formulae that change are those depending on the effective signature $s_{\text {eff }}=s \tilde{\Omega}$, which become

$$
\begin{aligned}
& \left\{K_{a}, K_{b}\right\}=s_{\text {eff }} \epsilon_{a b c} J^{c} \\
& \left\{K_{a}, P_{0}\right\}=-s_{\text {eff }} P_{a} .
\end{aligned}
$$

By redefining the signature $s$ to the correct Euclidean value $s=-1$ they can be restored to their previous form (14), (18) but with the Wick-rotated $\tilde{\Omega}$. Namely, applying $P_{0} \longrightarrow-i P_{0}$ to the Lorentzian deformation factor 
(24) we obtain its Euclidean counterpart

$$
\tilde{\Omega}^{E}=-\frac{P_{0}^{2}+\alpha}{\mathbf{P}^{2}-\alpha}
$$

and therefore the effective signature turns into $s_{\text {eff }}=$ $-\tilde{\Omega}^{E}$. Then the brackets (12,20) define the deformed Euclidean algebra, while in the low energy limit $s \tilde{\Omega}^{E}(0,0) \rightarrow$ -1 and hence the classical Euclidean algebra is recovered.

Furthermore, Wick-rotating the Lorentzian Casimir element (23) we obtain

$$
\mathcal{C}_{1}^{E}=\frac{P_{0}^{2}+\mathbf{P}^{2}}{1-\alpha^{-1} \mathbf{P}^{2}} .
$$

One can observe that for $\alpha<0$ the expression (52) is a positive definite function, while for $\alpha>0$ it is positive below the invariant energy scale $|\mathbf{P}|<\sqrt{\alpha}$, negative above this scale $|\mathbf{P}|>\sqrt{\alpha}$ and divergent at $|\mathbf{P}|=\sqrt{\alpha}$. The negativity of $\mathcal{C}_{1}^{E}$ can indicate that we are entering the hyperbolic regime of the Laplace operator. However, this is so only if the relation between the Laplace operator and the mass Casimir is linear, while in general this may not be the case.

In order to explore the other possible definition of the Euclidean counterpart of our model we first assume that $s=-1$. In this case the classical limit gives us the condition $s \tilde{\Omega}^{E}(0,0)=-1$, equivalent to $\tilde{\Omega}^{E}(0,0)=1$. The latter is the same as in the Lorentzian case for the function $\tilde{\Omega}$. Consequently, the solution for $\tilde{\Omega}^{E}$ is analogous to (21) and reads

$$
\tilde{\Omega}^{E}\left(P_{0}, \mathbf{P}^{2}\right)=\frac{P_{0}^{2} / c_{1}-1}{\mathbf{P}^{2} / c_{2}-1} .
$$

The agreement with the expression (51) can be achieved by setting $c_{2}=-c_{1}=\alpha$, for which (53) reduces to

$$
\tilde{\Omega}^{E}=-\frac{P_{0}^{2}+\alpha}{\mathbf{P}^{2}-\alpha} .
$$

Then the mass Casimir is again given by a superposition of $\tilde{\Omega}^{E}$ and the unit element, namely

$$
\mathcal{C}_{1}^{E}=a_{1} \tilde{\Omega}^{E}+a_{2} \mathbb{I}=\frac{a_{1} P_{0}^{2}+a_{2} \mathbf{P}^{2}+\alpha\left(a_{1}-a_{2}\right)}{\mathbf{P}^{2}-\alpha} .
$$

The conditions that guarantee the proper classical limit $\mathcal{C}_{1}^{E}=P_{0}^{2}+\mathbf{P}^{2}$ are $a_{2}=-a_{1}$ and $a_{1}=\alpha$, leading to

$$
\mathcal{C}_{1}^{E}=\frac{P_{0}^{2}+\mathbf{P}^{2}}{1-\alpha^{-1} \mathbf{P}^{2}}
$$

which is identical to (52), as it should.

\section{DIFFUSION AND THE SPECTRAL DIMENSION}

We have already collected all necessary ingredients to address the main subject of this paper - a diffusion process on (Wick-rotated) spacetime with the deformed symmetries (12]20). Namely, on a Riemannian manifold of $d$ topological dimensions and with the metric $g$ a diffusion (or random walk) is described by the heat equation

$$
\frac{\partial}{\partial \sigma} K\left(x, x_{0} ; \sigma\right)=\Delta_{x} K\left(x, x_{0} ; \sigma\right),
$$

where $\sigma$ denotes the auxiliary time (which plays the role of a scale parameter $), \Delta_{x}$ is the Laplacian and we assume the initial condition

$$
K\left(x, x_{0} ; \sigma=0\right)=\frac{\delta^{(d)}\left(x-x_{0}\right)}{\sqrt{|\operatorname{det} g(x)|}} .
$$

In general, the Laplacian may differ from the usual one $\Delta=g^{\mu \nu} \partial_{\mu} \partial_{\nu}, \mu, \nu=1, \ldots, d$. For $\mathbb{R}^{4}$ with the standard Euclidean metric the solution to (57), also called the heat kernel, can be written as

$$
K\left(x, x_{0} ; \sigma\right)=\int \frac{d^{4} P}{(2 \pi)^{4}} e^{i P_{\mu}\left(x-x_{0}\right)^{\mu}} e^{\sigma \Delta_{P}},
$$

where $\Delta_{P}$ is the Laplacian represented on space of momenta $P$. As one can see, the expression (59) is obtained using the Fourier transform.

For the model discussed in Sec. II we have shown that the deformed Poincaré algebra (12,20) can be consistently complemented by the undeformed Heisenberg algebra (25) (although it is not the unique choice). Then phase space has the ordinary structure and it seems that the usual notion of the Fourier transform should be preserved. However, in the case of models characterized by non-trivial phase space the Fourier transform can be substantially different from the standard one [34]. In particular, it may lead to a modification of the measure on momentum space, as it happens for the $\kappa$-Poincaré algebra 35. By analogy, the deformed measure (46) from Sec. IV can perhaps be coming from the corresponding modification of the Fourier transform, which arises due to the deformation of symmetries (12,20), irrespective of the form of the Heisenberg algebra.

In order to correctly define the counterpart of the heat kernel (59) for the considered model we have to make two changes that we have already suggested. Namely, use the appropriate Laplace operator $\Delta_{P}$ and the invariant measure on momentum space $d \mu(P)$. The invariance is necessary to guarantee the independence of the results from a particular reference frame, which has to be fulfilled at the level of the average return probability

$$
\begin{aligned}
P(\sigma) & :=\operatorname{tr} K(\sigma)=\frac{\int \sqrt{|\operatorname{det} g|} d^{4} x K(x, x ; \sigma)}{\int \sqrt{|\operatorname{det} g|} d^{4} x} \\
& =\int d \mu(P) e^{\sigma \Delta_{P}},
\end{aligned}
$$

obtained by the space averaging of the return probability $K(x, x ; \sigma)$. Both $\Delta_{P}$ and $d \mu(P)$ should be invariant quantities, leading to the invariance of the whole expression. Then the spectral dimension is defined as

$$
d_{S}(\sigma):=-2 \frac{\partial \log P(\sigma)}{\partial \log \sigma}
$$


and is a function of the scale parameter $\sigma$. Nevertheless, in our case $g$ is the Euclidean metric and hence $P(\sigma)=$ $K(x, x ; \sigma)$.

The invariance of the Laplace operator $\Delta_{P}$ under rotations and deformed boosts is guaranteed by the fact that it can be expressed in terms of the Euclidean mass Casimir as

$$
\Delta_{P}=-\mathcal{C}_{1}^{E}+\sum_{n=2} c_{n} \alpha\left(\frac{\mathcal{C}_{1}^{E}}{\alpha}\right)^{n} .
$$

The last term has been included to show that, in general, higher order powers of the Casimir may contribute to the expression for $\Delta_{P}$. However, here we restrict to the simplest possibility, setting $\forall_{n \geq 2} c_{n}=0$, simply due to the fact that we do not know what the extra $c_{n}$ parameters could be.

Taking $\Delta_{P}=-\mathcal{C}_{1}^{E}$ given by (56) and the invariant measure (46) we can finally write the following average return probability:

$$
P(\sigma)=\frac{4 \pi}{(2 \pi)^{4}} \int_{I_{p}} \frac{d p p^{2}}{\left(1-\frac{p^{2}}{\alpha}\right)^{3}} \int_{I_{E}} d E e^{-\sigma \frac{E^{2}+p^{2}}{1-p^{2} / \alpha}} .
$$

Here we introduce the notation $E \equiv P_{0}$ and $p \equiv|\mathbf{P}|$, while $I_{E}$ and $I_{p}$ denote the corresponding ranges of integration. The above expression can be given the probabilistic interpretation when $P(\sigma)>0$. It also has the correct classical limit

$$
\lim _{|\alpha| \rightarrow \infty} P(\sigma)=\frac{1}{16 \pi^{2}} \frac{1}{\sigma^{2}},
$$

for which one obtains $d_{S}(\sigma)=4$.

In the remaining part of this Section we will calculate the explicit form of $P(\sigma)$ with either sign of the parameter $\alpha$ and the appropriate intervals $I_{E}$ and $I_{p}$. From each of the results we will extract the corresponding spectral dimension (61).

A. The case $\alpha>0, I_{p}=[0, \sqrt{\alpha}], I_{E}=\mathbb{R}$

At the beginning let us consider two cases with positive $\alpha$. As we discussed in Sec. III then $\pm \sqrt{\alpha}$ are the invariant energy scales. Furthermore, at $p=\sqrt{\alpha}$ (note that $p>0)$ the Laplace operator $\Delta_{P}$ changes its type from elliptic to hyperbolic. For this reason the integration range of $p$ has to be restricted to $I_{p}=[0, \sqrt{\alpha}]$. On the other hand, crossing of the value $E=\sqrt{\alpha}$ does not change sign of the mass Casimir (52) and therefore we keep here the full energy range $I_{E}=\mathbb{R}$. A case with the bounded $I_{E}$ will be discussed in the next Subsection. Applying the above integration intervals to (63) we obtain the average return probability

$$
\begin{aligned}
P(\sigma) & =\frac{4 \pi}{(2 \pi)^{4}} \sqrt{\frac{\pi}{\sigma}} \int_{0}^{\sqrt{\alpha}} \frac{d p p^{2} e^{-\sigma \frac{p^{2}}{1-p^{2} / \alpha}}}{\left(1-\frac{p^{2}}{\alpha}\right)^{5 / 2}} \\
& =\frac{4 \pi}{(2 \pi)^{4}} \sqrt{\frac{\pi}{\sigma}} \int_{0}^{\infty} d q q^{2} e^{-\sigma q^{2}} \\
& =\frac{1}{16 \pi^{2}} \frac{1}{\sigma^{2}}
\end{aligned}
$$

where the change of variables to $q:=\frac{p}{\sqrt{1-p^{2} / \alpha}}$ has converted the seemingly complicated formula into the case (64) of Euclidean space with the ordinary Laplacian. Substituting this result into (61) we calculate the spectral dimension

$$
d_{S}(\sigma)=4
$$

Therefore, for $\alpha<0$ and the integration over momentum space restricted to $I_{p}=[0, \sqrt{\alpha}]$, we measure the same dimension of (Wick-rotated) spacetime as for the four-dimensional Minkowski spacetime. However, this not necessarily means that the two spaces are isomorphic to each other.

\section{B. The case $\alpha>0, I_{p}=[0, \sqrt{\alpha}], I_{E}=[-\sqrt{\alpha}, \sqrt{\alpha}]$}

Another possibility is that, in contrast to the previous Subsection, energy is restricted to the range between the invariant scales, i.e. $I_{E}=[-\sqrt{\alpha}, \sqrt{\alpha}]$. Then, performing the calculations analogously to (65), we find that (63) has the form

$$
P(\sigma)=\frac{4 \pi}{(2 \pi)^{4}} \sqrt{\frac{\pi}{\sigma}} \int_{0}^{\infty} d q q^{2} e^{-\sigma q^{2}} \operatorname{erf}\left(\sqrt{\sigma\left(q^{2}+\alpha\right)}\right),
$$

with the following asymptotic behaviors:

$$
P(\sigma) \sim \sigma^{-2} \text { for } \sigma \rightarrow \infty,
$$

and

$$
P(\sigma) \sim \sigma^{-2} \text { for } \quad \sigma \rightarrow 0 .
$$

The spectral dimension corresponding to (67) can only be obtained numerically and its running is shown in Fig. 1. Curiously, the restriction of energy to $[-\sqrt{\alpha}, \sqrt{\alpha}]$ leads here to a slight deviation from $d_{S}=4$ at intermediate diffusion times $\sigma \sim 1 / \alpha$, characterized by the invariant energy scale. In principle we could also consider the cases with $I_{E}=[-\infty,-\sqrt{\alpha}]$ or $I_{E}=[\sqrt{\alpha}, \infty]$. However, this would not allow us to probe the large scale limit of spacetime, which is associated with low energy contributions to the Laplace operator. 


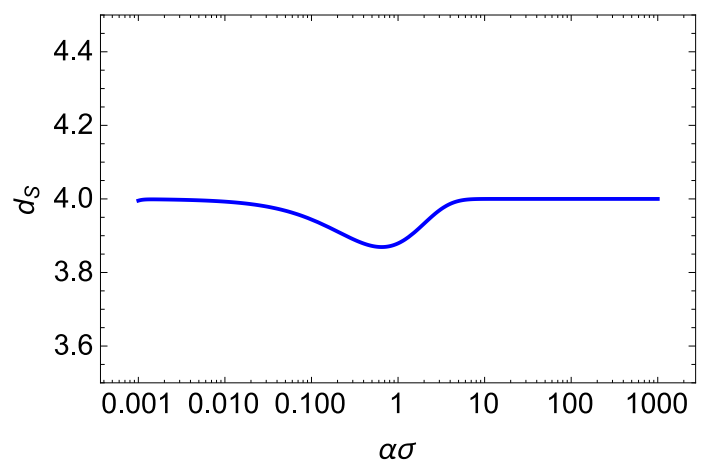

FIG. 1. Spectral dimension as a function of the scale parameter for the case with $\alpha>0$ and $I_{E}=[-\sqrt{\alpha}, \sqrt{\alpha}]$.

\section{The case $\alpha<0, I_{p}=\mathbb{R}, I_{E}=\mathbb{R}$}

Let us now turn to the case of $\alpha<0$. For the Lorentzian signature $s=1$ the sign of $\tilde{\Omega}$ remains constant irrespective of the values of $E$ and $p$, and hence the signature change does not occur. In turn, the Euclidean $\tilde{\Omega}^{E}$ changes its sign at $P_{0}^{2}=-\alpha$. Nevertheless, the Laplace operator $\Delta_{P}$ remains elliptic in the whole range of $E$ and $p$ and we have no reason to restrict the intervals $I_{E}$ and $I_{p}$. Similarly, there is no divergence in either $\tilde{\Omega}^{E}$ or $\Delta_{P}$ and, which will turn out to be essential, the value of $\tilde{\Omega}^{E}$ tends to zero in the $\mathbf{P} \rightarrow \infty$ limit.

Integrating (63) over $E$ and making the change of variables to $u:=\frac{p}{\sqrt{1+p^{2} /|\alpha|}}$ we obtain

$$
\begin{aligned}
P(\sigma) & =\frac{4 \pi}{(2 \pi)^{4}} \sqrt{\frac{\pi}{\sigma}} \int_{0}^{\infty} \frac{d p p^{2} e^{-\sigma \frac{p^{2}}{1+p^{2} /|\alpha|}}}{\left(1+\frac{p^{2}}{|\alpha|}\right)^{5 / 2}} \\
& =\frac{4 \pi}{(2 \pi)^{4}} \sqrt{\frac{\pi}{\sigma}} \int_{0}^{\sqrt{|\alpha|}} d u u^{2} e^{-\sigma u^{2}} \\
& =\frac{\operatorname{erf}(\sqrt{|\alpha| \sigma})}{16 \pi^{2} \sigma^{2}}-\frac{e^{-|\alpha| \sigma} \sqrt{|\alpha|}}{8 \pi^{5 / 2} \sigma^{3 / 2}}
\end{aligned}
$$

which has the asymptotic behaviors:

$$
P(\sigma) \sim \sigma^{-2} \text { for } \sigma \rightarrow \infty
$$

and

$$
P(\sigma) \sim \sigma^{-1 / 2} \text { for } \quad \sigma \rightarrow 0 .
$$

The analytic expression for the running spectral dimension calculated from (70) is

$$
d_{S}(\sigma)=4-\frac{4(|\alpha| \sigma)^{3 / 2}}{\sqrt{\pi} e^{|\alpha| \sigma} \operatorname{erf}(\sqrt{|\alpha| \sigma})-2 \sqrt{|\alpha| \sigma}}
$$

and the corresponding plot is presented in Fig. 2 .

We observe here the dimensional reduction from the large scale value $d_{S}=4$ to $d_{S}=1$ at small scales. The

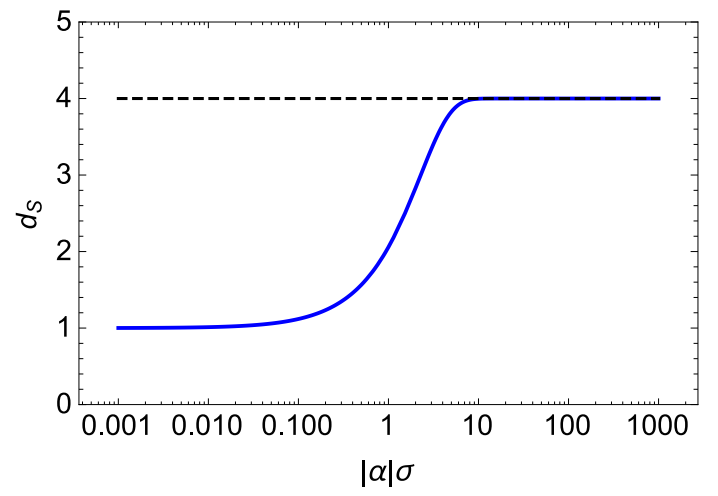

FIG. 2. Spectral dimension as a function of the scale parameter for the case with $\alpha<0$ with $I_{E}=\mathbb{R}$.

latter ultraviolet value can have the following interpretation. At high energies $|\mathbf{P}| \rightarrow \infty$ the deformation factor $\tilde{\Omega}$ tends to zero, corresponding to the so-called ultralocal [36] or Carrollian limit 37, 38] of spacetime and its relativistic symmetries. In the Carrollian limit, in which the speed of light is taken to zero, spacelike separated points become effectively decoupled, due to the collapse of their lightcones into null worldlines. Then spacetime becomes a congruence of such (one-dimensional) worldlines, as it is schematically depicted in Fig. 3. Since increasing energy is equivalent to probing smaller scales of spacetime, we indeed have the dimensional reduction to $d_{S}=1$. Such a value is consistent with the analysis [26] of loop quantum cosmology in the case characterized by the hypersurface deformation algebra (1],3). The significance of these results is that ultralocality is one of the features of the Belinsky-Khalatnikov-Lifshitz (BKL) conjecture [39] or asymptotic silence scenario.

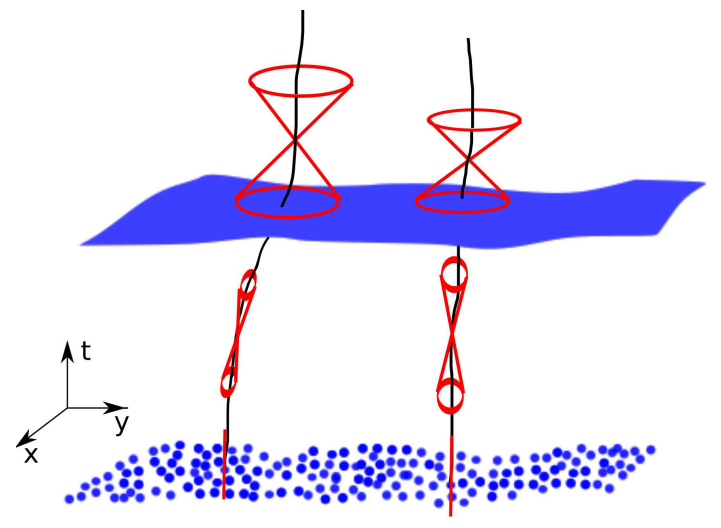

FIG. 3. Schematic representation of the collapse of lightcones into a congruence of null worldlines while approaching the ultralocal (silent) state.

On the other hand, in [8] it has been argued that asymptotic silence is characterized by the dimensional reduction to 2 , which results from an elongation of the anisotropic cosmological model in one particular direction during each of the Kasner epochs. Then spacetime 
effectively has one spatial and one temporal direction. However, such a viewpoint is in contradiction with ultralocality, which is achieved first in the BKL scenario. Namely, in this scenario universe first decouples into noninteracting points of space, each described by the minisuperspace homogeneous cosmological model. Then the chaotic dynamics is acting at the level of the points. Treating an elongation in the internal space of a point as an elongation in the three-dimensional space violates the initial suppression of the spatial dependence of fields. Therefore, the interpretation in which the BKL conjecture leads to the dimensional reduction to 1 seems to be better justified.

It should be stressed that the results in this Subsection (as well as in the previous ones) have been obtained in the Euclidean domain of the model, which can be treated either as an independent case or as the Wick-rotated version of the Lorentzian model. The second possibility is supported by the observed consistency with the Lorentzian picture of the collapse of lightcones. Therefore, we conclude that the dimensional reduction of our model does not fundamentally depend on the spacetime metric signature. This is also in agreement with the fact that, irrespective of the signature $s$, in the limit $\tilde{\Omega} \rightarrow 0\left(\right.$ or $\left.\tilde{\Omega}^{E} \rightarrow 0\right)$ the effective signature $s_{\text {eff }}=s \tilde{\Omega}$ (or $s_{\text {eff }}=s \tilde{\Omega}^{E}$ ) tends to zero and the deformed algebra (12]20) becomes the (standard) Carroll algebra.

\section{The case $\alpha<0, I_{p}=\mathbb{R}, I_{E}=[-\sqrt{\alpha}, \sqrt{\alpha}]$}

While the Laplace operator for $\alpha<0$ is elliptic independently of the value of $P_{0}$, in order to make our discussion complete we will also consider here the restriction to $I_{E}=[-\sqrt{\alpha}, \sqrt{\alpha}$. The motivation for taking into account such a range of variability of $P_{0}$ is the changing

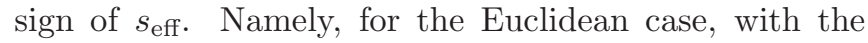
deformation factor (51), we find that

$$
s_{\mathrm{eff}}=-\tilde{\Omega}^{E}=\frac{P_{0}^{2}+\alpha}{\mathbf{P}^{2}-\alpha} .
$$

For $\alpha<0$ the denominator remains positive definite and the effective signature $s_{\text {eff }}$ is negative (Euclidean) for $P_{0}^{2}<|\alpha|$, while for $P_{0}^{2}=|\alpha|$ we obtain $s_{\text {eff }}=0$ and for $P_{0}^{2}>|\alpha|$ the signature $s_{\text {eff }}$ becomes positive (Lorentzian). Therefore, while entering the $P_{0}^{2}>|\alpha|$ region does not affect the elliptic character of the Laplace operator, the effective signature becomes Lorentzian. Below we make an analysis of the diffusion process assuming that the $P_{0}^{2}>|\alpha|$ regime is excluded from the physical phase space. In other words, a UV cut-off is introduced at the energy scale $P_{0}=\sqrt{|\alpha|}$.

In this case the average return probability can be written as

$$
P(\sigma)=\frac{|\alpha|^{2} e^{-\frac{\tau}{2}}}{16 \pi^{2} \tau} \int_{-1}^{1} \frac{d x e^{-\tau x^{2} / 2}}{1-x^{2}} \mathrm{I}_{1}\left(\frac{\tau}{2}\left(1-x^{2}\right)\right),
$$

where $\tau:=\sigma|\alpha|$ and $\mathrm{I}_{1}(x)$ denotes a Bessel function. The expression (75) has the following asymptotic behaviors:

$$
P(\sigma) \sim \sigma^{-2} \text { for } \sigma \rightarrow \infty,
$$

and

$$
P(\sigma) \sim \text { const for } \sigma \rightarrow 0 .
$$

The UV behavior suggests that the spectral dimension reduces to $d_{S}=0$ in this limit. This expectation is supported by calculating $d_{S}$ as a function of $\sigma$ with the use of the definition (61). The resulting $d_{S}(\sigma)$ dependence is plotted in Fig. 4

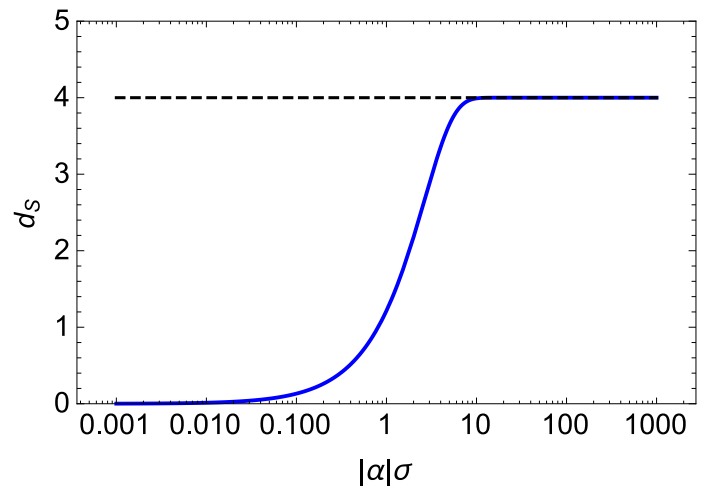

FIG. 4. Spectral dimension as a function of the scale parameter for the case with $\alpha<0$ with $I_{E}=[-\sqrt{\alpha}, \sqrt{\alpha}$.

How to understand such a result? In the case with $I_{E}=\mathbb{R}$ the spectral dimension in the UV limit reduces to $d_{S}=1$, which, as we already discussed, can be associated with the time direction of spacetime. Introducing the cut-off $\sqrt{|\alpha|}$ in the energy domain (which is the case discussed in the present Subsection) prevents the probing of the time direction at time scales smaller than $1 / \sqrt{|\alpha|}$. In other words, when the energy cut-off is introduced, time effectively becomes discrete and undefined at the scales smaller than $1 / \sqrt{|\alpha|}$. Therefore, it is natural to expect that the time dimension (which remains physically available in the ultralocal case) disappears at sufficiently short time scales if the energy cut-off is introduced.

\section{SUMMARY}

The analysis presented in this paper addressed the issue of probing a loop-quantized spacetime configuration by the diffusion process. The considered model was given by Minkowski spacetime but with symmetries described by a deformed Poincaré algebra, whose form is motivated by the predictions of the effective regime of loop quantum gravity. While such an algebra is still unknown in general, we proposed a specific case that has the structure recovered from the loop-deformed hypersurface deformation algebra. Namely, using reasonable assumptions, like the conservation of the Jacobi identities by the deformed 
Poincaré algebra, we have determined the particular form of the deformation factor. We also assumed an extension of such a symmetry algebra by the standard Heisenberg algebra of phase space variables.

Subsequently we showed that our symmetry algebra leads to deformed Lorentz transformations, which preserve two distinguished energy scales. Moreover, we have derived the form of the invariant measure on momentum space. The above ingredients were applied to precisely define (after the Wick rotation) and analyze the diffusion process on spacetime endowed with the considered deformed algebra of symmetries. In particular, we have found that in the case in which the deformation factor tends to zero in the high energy limit, the spectral dimension reduces to $d_{S}=1$ at small scales, as it is expected in the asymptotic silence scenario. Besides, the presence of the invariant energy scale allows to consider our model as an example of the doubly special relativity (DSR), which could be further studied within the latter framework.

The UV values of the spectral dimension for all the cases considered in this article are collected in the table below.

\begin{tabular}{|c|c|c|c|c|}
\hline Case & $\alpha$ & $I_{p}$ & $I_{E}$ & $d_{S}(\sigma \rightarrow 0)$ \\
\hline $\mathrm{A}$ & $>0$ & {$[0, \sqrt{\alpha}]$} & $\mathbb{R}$ & 4 \\
$\mathrm{~B}$ & $>0$ & {$[0, \sqrt{\alpha}]$} & {$[-\sqrt{\alpha}, \sqrt{\alpha}]$} & 4 \\
$\mathrm{C}$ & $<0$ & $\mathbb{R}$ & $\mathbb{R}$ & 1 \\
$\mathrm{D}$ & $<0$ & $\mathbb{R}$ & {$[-\sqrt{\alpha}, \sqrt{\alpha}]$} & 0 \\
\hline
\end{tabular}

Finally, let us make a brief comparison of our derivation of the deformed Poincaré algebra with the LQG derivation in the spherically symmetric case in [19, 20]. In this paper we have begun with assuming a general deformation of the bracket (3), parametrized by some unknown function $\Omega$ of phase space variables. As it should be stressed, the subsequent reduction of the DHDA to the corresponding deformed Poincaré algebra requires the use of a certain averaging procedure. The obtained deformation of the Poincaré algebra is then parametrized by the $s_{\text {eff }}$ function, whose form is inferred from the consistent mathematical assumptions rather than some known physical solution for $\Omega$. The family of functions $s_{\text {eff }}$ considered in this paper does not cover all possible choices of $\Omega$. Moreover, it is not yet known if the discussed form of $s_{\text {eff }}$ can be associated with the cosine form of $\Omega$ that appears in the LQG models with holonomy corrections. In contrast, in [19, 20] the cosine form of $\Omega$ is suggested by the presence of the calculated holonomy corrections. It is subsequently assumed that the corresponding deformation factor of the Poincaré algebra is of the same cosine form. However, as we have to stress, a straightforward identification of the form of HDA deformation function $\Omega$ with the corresponding deformation of the Poincaré algebra is not necessarily correct and the known results in the spherically symmetric case need to be confirmed by the further analysis. Due to such differences between both of the discussed approaches, at the moment we are only able to observe the qualitative but not the quantitative similarities between their results.

\section{ACKNOWLEDGEMENTS.}

We thank Anna Pachoł for very useful discussions. This work is supported by the Iuventus Plus grant No. 0302/IP3/2015/73 from the Polish Ministry of Science and Higher Education. TT was additionally supported by the National Science Centre Poland, project 2014/13/B/ST2/04043.

\section{APPENDIX}

The aim of this Appendix is to show explicitly how the classical hypersurface deformation algebra reduces to the Poincaré or Euclidean algebra, depending on the metric signature $s$. A more detailed discussion of these calculations can be found e.g. in [40].

The hypersurface deformation algebra describes deformations of an arbitrary spatial hypersurface $\Sigma_{t}$, which correspond to local diffeomorphisms. The generators of a deformation are the smeared scalar constraint $S[N]$ and the smeared spatial diffeomorphisms constraint $D\left[N^{a}\right]$. $S[N]$ generates deformations in the direction normal to the hypersurface $\Sigma_{t}$ (i.e. the time direction), while $D\left[N^{a}\right]$ is responsible for deformations in the tangential direction. The deformations are parametrized by a lapse function $N$ and shift vector $N^{a}$, which together form the deformation vector $u^{\mu}=N n^{\mu}+N^{\mu}$, where $n^{\mu}$ is a unit vector normal to the hypersurface $\Sigma_{t}$, such that $g_{\mu \nu} n^{\mu} n^{\nu}=s$. Here $\mu, \nu=0,1,2,3$ denote spacetime indices and $a, b=1,2,3$ the spatial ones. The local coordinate transformation generated by $u^{\mu}$ is given by $x^{\mu} \rightarrow x^{\mu}=x^{\mu}+u^{\mu}$.

The constraints $S[N]$ and $D\left[N^{a}\right]$ are contributions to the gravitational Hamiltonian in the ADM formulation, $H\left[N, N^{a}\right]=S[N]+D\left[N^{a}\right]$. Furthermore, they form the first class algebra:

$$
\begin{aligned}
\left\{D\left[N_{1}^{a}\right], D\left[N_{2}^{a}\right]\right\} & =D\left[N_{1}^{b} \partial_{b} N_{2}^{a}-N_{2}^{b} \partial_{b} N_{1}^{a}\right] \\
\left\{S[N], D\left[N^{a}\right]\right\} & =-S\left[N^{b} \partial_{b} N\right] \\
\left\{S\left[N_{1}\right], S\left[N_{2}\right]\right\} & =D\left[s q^{a b}\left(N_{1} \partial_{b} N_{2}-N_{2} \partial_{b} N_{1}\right)\right] .
\end{aligned}
$$

The above algebra is satisfied by any theory that is covariant under local diffeomorphisms. Let us note that due to the presence of the spatial metric $q^{a b}$ in the last line the brackets (78, 80) do not define a Lie algebra.

A special class of local diffeomorphisms are linear transformations, which are associated with the Poincaré (or respectively Euclidean) symmetry. The corresponding brackets of the symmetry generators can be recovered from the hypersurface deformation algebra 78,80 by restricting the (infinitesimal) deformations to linear 
functions, such that we have

$$
\begin{aligned}
N(x) & =\Delta t+v_{a} x^{a}, \\
N^{a}(x) & =\Delta x^{a}+R^{a}{ }_{b} x^{b}, \\
q_{a b} & =\delta_{a b},
\end{aligned}
$$

where $\Delta t$ is the parameter of a time translation, $v_{a}$ parametrize boosts, $\Delta x^{a}$ specify spatial translations and $R^{a}{ }_{b}$ is a rotation matrix. The rotation matrix in terms of infinitesimal angles $\varphi^{a}$ is given by $R^{a b}=\epsilon^{b a c} \varphi_{c}$.

In the linear case (81, 83) the constraints $D\left[N^{a}\right]$ and $S[N]$ can be expressed as the following combinations of the Poincaré (or respectively Euclidean) generators:

$$
\begin{aligned}
D\left[N^{a}\right] & =-\Delta x^{a} P_{a}-\varphi^{a} J_{a}, \\
S[N] & =-\Delta t P_{0}-v^{a} K_{a},
\end{aligned}
$$

where $P_{0}$ is the generator of time translations, $P_{a}$ are the generators of spatial translations, $J_{a}$ generators of rotations and $K_{a}$ generators of boosts. The minus signs are necessary to obtain the symmetry generators in the usual convention. Then the left hand side of the bracket (78) can be rewritten as

$$
\begin{aligned}
\left\{D\left[N_{1}^{a}\right], D\left[N_{2}^{a}\right]\right\} & =\left\{\Delta x_{1}^{a} P_{a}+\varphi_{1}^{a} J_{a}, \Delta x_{1}^{b} P_{b}+\varphi_{1}^{b} J_{b}\right\} \\
& =\Delta x_{1}^{a} \Delta x_{2}^{b}\left\{P_{a}, P_{b}\right\} \\
& +\left(\varphi_{1}^{a} \Delta x_{2}^{b}-\varphi_{2}^{a} \Delta x_{1}^{b}\right)\left\{J_{a}, P_{b}\right\} \\
& +\varphi_{1}^{a} \varphi_{2}^{b}\left\{J_{a}, J_{b}\right\}
\end{aligned}
$$

whereas the right hand side simplifies to

$$
\begin{aligned}
& D\left[N_{1}^{b} \partial_{b} N_{2}^{a}-N_{2}^{b} \partial_{b} N_{1}^{a}\right]= \\
& =D\left[\left(\Delta x_{1}^{b} \varphi_{2 c}-\Delta x_{2}^{b} \varphi_{1 c}\right) \epsilon^{a c b}-\epsilon^{a b c}\left(\epsilon_{b d e} \varphi_{1}^{d} \varphi_{2}^{e}\right) x_{c}\right] \\
& =\left(\Delta x_{2}^{b} \varphi_{1 c}-\Delta x_{1}^{b} \varphi_{2 c}\right) \epsilon^{a c b} P_{a}+\epsilon^{b d e} \varphi_{1 d} \varphi_{2 e} J_{b} .
\end{aligned}
$$

The final expressions in (86) and (87) agree with (78) if and only if the appropriate terms on both sides (which are multiplied by parameters of the deformations) are equal to each other. This condition gives us the first three brackets of the Poncaré algebra:

$$
\begin{aligned}
& \left\{P_{a}, P_{b}\right\}=0, \\
& \left\{J_{a}, P_{b}\right\}=\epsilon_{a b c} P^{c}, \\
& \left\{J_{a}, J_{b}\right\}=\epsilon_{a b c} J^{c} .
\end{aligned}
$$

The analogous procedure can now be applied to the bracket (79). At the left hand side we calculate

$$
\begin{aligned}
& \left\{S[N], D\left[N^{a}\right]\right\}=\left\{\Delta t P_{0}+v^{a} K_{a}, \Delta x^{b} P_{b}+\varphi^{b} J_{b}\right\} \\
& =\Delta t \Delta x^{b}\left\{P_{0}, P_{b}\right\}+\Delta t \varphi^{b}\left\{P_{0}, J_{b}\right\} \\
& +v^{a} \Delta x^{b}\left\{K_{a}, P_{b}\right\}+v^{a} \varphi^{b}\left\{K_{a}, J_{b}\right\}
\end{aligned}
$$

while at the right hand side we have

$$
\begin{aligned}
& -S\left[N^{a} \partial_{a} N\right]=-S\left[\Delta x^{a} v_{a}+v^{a} R_{b}^{a} x^{b}\right] \\
& =\Delta x^{a} v_{a} P_{0}-v^{a} \epsilon^{a b c} \varphi_{c} K^{b} .
\end{aligned}
$$

Comparing (91) with (92) we obtain the brackets:

$$
\begin{aligned}
\left\{P_{0}, P_{a}\right\} & =0, \\
\left\{P_{0}, J_{a}\right\} & =0, \\
\left\{K_{a}, P_{b}\right\} & =\delta_{a b} P_{0}, \\
\left\{J_{a}, K_{b}\right\} & =\epsilon^{a b c} K_{c} .
\end{aligned}
$$

Finally, let us consider the bracket (80). In this case the left hand side gives

$$
\begin{aligned}
& \left\{S\left[N_{1}\right], S\left[N_{2}\right]\right\}=\left\{\Delta t_{1} P_{0}+v_{1}^{a} K_{a}, \Delta t_{2} P_{0}+v_{2}^{b} K_{b}\right\} \\
& =\Delta t_{1} \Delta t_{2}\left\{P_{0}, P_{0}\right\}+\left(v_{1}^{a} \Delta t_{2}-v_{1}^{a} \Delta t_{1}\right)\left\{K_{a}, P_{0}\right\} \\
& +v_{1}^{a} v_{2}^{b}\left\{K_{a}, K_{b}\right\}
\end{aligned}
$$

and the right hand side

$$
\begin{aligned}
& D\left[s q^{a b}\left(N_{1} \partial_{b} N_{2}-N_{2} \partial_{b} N_{1}\right)\right] \\
& =s D\left[\left(v_{1}^{a} \Delta t_{1}-v_{1}^{a} \Delta t_{2}\right)+\epsilon^{a b c}\left(\epsilon_{b d e} v_{1}^{d} v_{2}^{e}\right) x^{c}\right] \\
& =-s\left(v_{1}^{a} \Delta t_{1}-v_{1}^{a} \Delta t_{2}\right) P_{a}-s \epsilon_{a b c} v_{1}^{b} v_{2}^{c} J^{a},
\end{aligned}
$$

which leads to

$$
\begin{aligned}
\left\{K_{a}, P_{0}\right\} & =-s P_{a}, \\
\left\{K_{a}, K_{b}\right\} & =-s \epsilon^{a b c} K_{c} .
\end{aligned}
$$

The latter brackets are the only ones which are affected by the signature $s$.

The obtained set of nine brackets (88, 90, (93, 96) and (99,100) defines the Poincaré (or respectively Euclidean) algebra.
[1] D. Nesterov and S. N. Solodukhin, Nucl. Phys. B 842, 141 (2011) arXiv:1007.1246 [hep-th]].

[2] J. Ambjorn, J. Jurkiewicz and R. Loll, Phys. Rev. Lett. 95, 171301 (2005) hep-th/0505113.
[3] D. N. Coumbe and J. Jurkiewicz, JHEP 1503, 151 (2015) arXiv:1411.7712 [hep-th]].

[4] S. Carlip, AIP Conf. Proc. 1196, 72 (2009) arXiv:0909.3329 [gr-qc]]. 
[5] P. Hořava, Phys. Rev. Lett. 102, 161301 (2009) arXiv:0902.3657 [hep-th]].

[6] O. Lauscher and M. Reuter, JHEP 0510, 050 (2005) hep-th/0508202.

[7] G. Calcagni, Phys. Rev. D 86, 044021 (2012) arXiv:1204.2550 [hep-th]].

[8] S. Carlip, Class. Quant. Grav. 32, 232001 (2015) arXiv:1506.08775 [gr-qc]].

[9] A. Belenchia, D. M. T. Benincasa, A. Marcianò and L. Modesto, Phys. Rev. D 93, 044017 (2016) arXiv:1507.00330 [gr-qc]].

[10] L. Modesto, Class. Quant. Grav. 26, 242002 (2009) arXiv:0812.2214 [gr-qc]].

[11] G. Calcagni, D. Oriti and J. Thürigen, Phys. Rev. D 91, 084047 (2015) arXiv:1412.8390 [hep-th]].

[12] D. Benedetti, Phys. Rev. Lett. 102, 111303 (2009) arXiv:0811.1396 [hep-th]].

[13] M. Arzano and T. Trześniewski, Phys. Rev. D 89, 124024 (2014) arXiv:1404.4762 [hep-th]].

[14] G. Amelino-Camelia, M. Arzano, G. Gubitosi and J. Magueijo, Phys. Lett. B 736, 317 (2014) arXiv:1311.3135 [gr-qc]]

[15] M. Bojowald and G. M. Paily, Phys. Rev. D 86, 104018 (2012) arXiv:1112.1899 [gr-qc]].

[16] T. Cailleteau, J. Mielczarek, A. Barrau and J. Grain, Class. Quant. Grav. 29, 095010 (2012) arXiv:1111.3535 [gr-qc]].

[17] M. Bojowald and J. Mielczarek, JCAP 1508, 052 (2015) arXiv:1503.09154 [gr-qc]].

[18] J. Mielczarek, Springer Proc. Phys. 157, 555 (2014) arXiv:1207.4657 [gr-qc]].

[19] M. Bojowald and G. M. Paily, Phys. Rev. D 87, 044044 (2013) arXiv:1212.4773 [gr-qc]].

[20] G. Amelino-Camelia, M. M. Da Silva, M. Ronco, L. Cesarini and O. M. Lecian, Phys. Rev. D 95, 024028 (2017) arXiv:1605.00497 [gr-qc]].

[21] S. Brahma, M. Ronco, G. Amelino-Camelia and A. Marcianò, Phys. Rev. D 95, 044005 (2017) arXiv:1610.07865 [gr-qc]].
[22] M. Ronco, Adv. High Energy Phys. 2016, 9897051 (2016) arXiv:1605.05979 [gr-qc]].

[23] G. Amelino-Camelia, J. Lukierski and A. Nowicki, Int. J. Mod. Phys. A 14, 4575 (1999) gr-qc/9903066.

[24] J. Mielczarek, Europhys. Lett. 108, 40003 (2014) arXiv:1304.2208 [gr-qc]].

[25] M. Bojowald, U. Büyükçam, S. Brahma and F. D'Ambrosio, Phys. Rev. D 94, 104032 (2016) arXiv:1610.08355 [gr-qc]].

[26] J. Mielczarek, AIP Conf. Proc. 1514, 81 (2012) arXiv:1212.3527 [gr-qc]].

[27] J. D. Brown and J. W. York, Jr., Phys. Rev. D 47, 1407 (1993) gr-qc/9209012.

[28] D. Kovačević, S. Meljanac, A. Pachoł and R. Štrajn, Phys. Lett. B 711, 122 (2012) arXiv:1202.3305 [hep-th]].

[29] A. Borowiec and A. Pachol, SIGMA 6, 086 (2010) arXiv:1005.4429 [math-ph]].

[30] F. Cianfrani, J. Kowalski-Glikman, D. Pranzetti and G. Rosati, Phys. Rev. D 94, 084044 (2016) arXiv:1606.03085 [hep-th]].

[31] J. Magueijo and L. Smolin, Phys. Rev. Lett. 88, 190403 (2002) hep-th/0112090.

[32] G. Calcagni, A. Eichhorn and F. Saueressig, Phys. Rev. D 87, 124028 (2013) arXiv:1304.7247 [hep-th]].

[33] J. Lukierski, H. Ruegg and A. Nowicki, J. Math. Phys. 35, 2607 (1994).

[34] C. Guedes, D. Oriti and M. Raasakka, J. Math. Phys. 54, 083508 (2013) arXiv:1301.7750 [math-ph]].

[35] L. Freidel, J. Kowalski-Glikman and S. Nowak, Int. J. Mod. Phys. A 23, 2687 (2008) arXiv:0706.3658 [hep-th]].

[36] C. J. Isham, Proc. Roy. Soc. Lond. A 351, 209 (1976).

[37] J.-M. Lévy-Leblond, Annales de l'I.H.P., section A, tome 3, no 1 (1965).

[38] H. Bacry and J.-M. Lévy-Leblond, J. Math. Phys. 9, 1605 (1968).

[39] V. A. Belinsky, I. M. Khalatnikov and E. M. Lifshitz, Adv. Phys. 19, 525 (1970).

[40] T. Thiemann, "Modern Canonical Quantum General Relativity," Cambridge University Press (2008). 